Check for updates

Cite this: RSC Adv., 2017, 7, 19976

Received 8th March 2017

Accepted 30th March 2017

DOI: $10.1039 / \mathrm{c} 7 \mathrm{ra02839c}$

rsc.li/rsc-advances

\section{Polymer brush decorated nanoparticles immobilised on polymer monoliths for enhanced biopolymer elution $\uparrow$}

\begin{abstract}
M. lacono, ${ }^{a}$ D. Connolly ${ }^{b}$ and A. Heise (D) *ac
A protocol for the decoration of a polymeric monolith with polymer brush grafted silica nanoparticles was developed. Monolithic poly(glycidyl methacrylate-co-ethylene glycol dimethacrylate) (GMA-CO-EDMA) was prepared in $15 \mathrm{~mm}$ long cylindrical polypropylene tubing and quarternized. $160 \mathrm{~nm}$ silica nanoparticles with a $20 \mathrm{~nm}$ poly(methacrylic acid-co-tert-butyl methacrylate) brush layer were flushed through the monolith and electrostatically trapped. A uniform and dense carboxylic acid containing brush nanoparticle surface decoration over the entire length of the monolith was achieved. Elution performance of the brush nanoparticle decorated monolith was compared to a carboxylic acid surface decorated monolith using myoglobin and blue dextran analytes. Quantitative analysis of critical elution parameters confirms an elution enhancement of the brush nanoparticle system, which is higher than expected from simple geometrical considerations of surface area increase and thus an effect of the polymer brush on the nanoparticle. The presented proof of concept offers a highly adaptable platform as polymer brushes of a broad spectrum (functional groups, hydrophilicity, etc.) are readily accessible offering the opportunity to tailor monolithic surfaces towards the extraction problem.
\end{abstract}

\section{Introduction}

Polymer monoliths are single continuous rods of porous material through which a mobile phase can readily flow at considerably reduced back-pressure. ${ }^{1}$ Monolithic polymers can be obtained by a templating approach in which predominantly free radical polymerisation of a monomer/cross-linker mixture is carried out in the presence of a pore generating medium. Several different techniques have been described in the literature for example the polymerization of high-internal phase emulsions (polyHIPE) $)^{2-4}$ or polymerization in the presence of a porogenic solvent, ${ }^{5-7}$ among others. $^{8}$ Notably, polymer monoliths have emerged as an alternative stationary phase to traditional particulate materials in separation science.9-14 Following the first practical application of rigid macroporous polymer monoliths in HPLC by Svec and Frechet in $1992,{ }^{15}$ these materials have found application for affinity chromatography, ${ }^{\mathbf{1 6}}$ the separation of small molecules, ${ }^{17,18}$ proteomics, ${ }^{19}$ ionexchange chromatography, ${ }^{20}$ gas chromatography ${ }^{21}$ and solid

\footnotetext{
${ }^{a}$ School of Chemical Sciences, Dublin City University, Glasnevin, Dublin 9, Ireland ${ }^{b}$ APC Ltd., Cherrywood Business Park, Loughlinstown, Dublin 2, Ireland

${ }^{c}$ Royal College of Surgeons in Ireland, Department of Pharmaceutical and Medicinal Chemistry, 123 St. Stephens Green, Dublin 2, Ireland. E-mail: andreasheise@rcsi.ie; Tel: +3534025198

$\dagger$ Electronic supplementary information (ESI) available: Experimental procedures, FTIR spectra, extraction conditions and curve fitting parameters. See DOI: 10.1039/c7ra02839c
}

phase extraction. ${ }^{22,23}$ Typically, these applications rely on the interaction of an analyte with functional groups on the monolithic surface and the introduction of surface functionalities is thus a significant consideration in the material development. The most common approach is the use of functional acrylic monomers in the monolith formulation. While this has been successful for some functional monomers, the disadvantage of this approach is the need for polymerisation condition optimization for each individual monomer combination. ${ }^{8}$ Moreover, the functional group density can significantly vary as functional monomers might be buried in the bulk of the material not available at the pore surface. One approach to overcome this is by polymer brush decorated monoliths whereby a (functional) polymer is grafted from the monolithic surface thereby significantly increasing the functional group density and homogeneity. ${ }^{24}$ This concept has been successfully demonstrated for different types of monoliths applying free radical, ${ }^{25,26}$ controlled radical $^{27-32}$ as well as ring-opening polymerization. $^{33}$

Another growing field of interest in separation science is the application of nanoparticles. ${ }^{34-37}$ The main purpose of stationary phase modification with nanoparticles includes the increase of the specific surface area and the surface functionalization. Modification of polymer monolith stationary phases with a range of nanomaterials has been reported. ${ }^{38,39}$ This includes polymer particles such as charged latex nanoparticles for the ion-exchange and capillary electro-chromatographic separation of small ions. ${ }^{\mathbf{4 0 - 4 4}}$ The first reported strategy by 
Hilder involved the fabrication of a charged monolithic copolymer, poly(butyl methacrylate-co-ethylene dimethacrylate-co-2acrylamido-2-methyl-1-propane-sulfonic acid), (BuMA-coEDMA-co-AMPS) which was then coated with $60 \mathrm{~nm}$ latex nanoparticles. ${ }^{40}$ The AMPS monomer was used to generate a negatively charged surface prior to electrostatic attachment of the aminated latex and the separation of seven monosaccharides in under 10 minutes was demonstrated. Subsequent efforts by the same research group explored methods to increase the surface loading of charged NPs (and thus the ionexchange capacity of the NP-modified monolith) by initial sulphonation of a neutral glycidyl methacrylate-co-ethylene glycol dimethacrylate monolith using sodium sulphite followed by electrostatic coating with aminated latex NPs. ${ }^{42}$

Based on simple geometric considerations of a denselypacked monolayer of nanoparticles the maximum surface area increase achievable is by factor $\pi$ (Fig. S8, ESI $\dagger$ ) and this would offer a very limited increase in surface functionalities. Here we propose the combination of the advantages of high functional group density of polymer brushes with the nanoparticle decoration approach. To the best of our knowledge this is the first time the modification of a polymer monolith with polymer brush decorated silica nanoparticles (SiNP) is demonstrated. Specifically, $160 \mathrm{~nm}$ SiNP were grafted with poly(tert-butyl methacrylate). Deprotection of the tert-butyl ester groups yielded poly(methacrylic acid) brush decorated SiNPs (PMA-SiNP). In this instance, we have immobilized the polymer brush decorated SiNP with a high functional group density on a polymer monolith and demonstrated significantly enhanced ionexchange capacity for selected proteins, relative to a monolith that is not nano-structured. While this provides a first proof-ofconcept, the proposed system is highly flexible as polymer brushes on SiNP can easily be produced with a large variety of functionalities from commercially available monomers to meet specific separation challenges.

\section{Results and discussion}

\section{Monolith decoration with polymer brush NP}

Monolithic poly(glycidyl methacrylate-co-ethylene glycol dimethacrylate) (GMA-co-EDMA) was prepared in $15 \mathrm{~mm}$ cylindrical polypropylene tubing (inner diameter $=2.0 \mathrm{~mm}$ ) as previously reported by us. ${ }^{45}$ The monolith showed the typical globular surface structure of clusters of globules and channels as depicted in Fig. 1A. Luer-lock connectors were tightly attached to both ends of the tubes to allow connection to a syringe pump for subsequent modification steps (Fig. 1B).

Polymer brush decorated SiNP were obtained by surface polymerization of tert-butyl methacrylate from $160 \mathrm{~nm}$ initiator decorated SiNP using activators regenerated by electron transfer atom transfer radical polymerization (ARGET ATRP) as depicted in Fig. 2. ${ }^{\mathbf{4 6 , 4 7}}$ This resulted in a $20 \mathrm{~nm}$ polymer brush layer determined by Dynamic Light Scattering (DLS see Fig. S3, $\uparrow$ SEM image see Fig. S4, ESI†). Subsequently the tert-butyl groups were removed using phosphoric acid as a mild deprotection agent (Fig. 2A), which resulted in about 50\% deprotection yield as determined by thermogravimetric analysis (TGA). ${ }^{45}$ The polymer
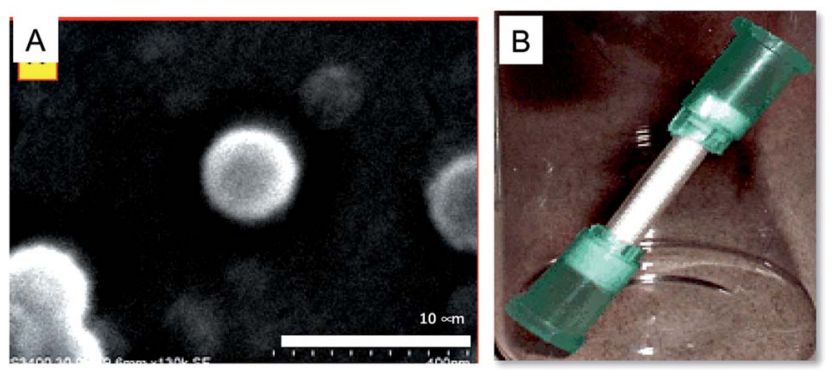

Fig. 1 (A) SEM image of a section of the GMA-Co-EDMA monolith showing the globules and macropores. (B) A $15 \mathrm{~mm}$ monolith in a polypropylene housing (inner diameter $2 \mathrm{~mm}$ ) with Luer-lock connectors.

brushes thus comprise a copolymer structure poly(tert-butyl acrylate-co-acrylic acid) but it is assumed that unprotected acrylic acid is only present close to the silica core as the particles were hydrophilic and fully suspendable in water (denoted as PMA-SiNP). ${ }^{47}$

The steps involved in the decoration of the monolith with the PMA-SiNP are depicted in Fig. 2B. In the first step the GMA epoxy groups of the monolith were reacted with trimethylamine

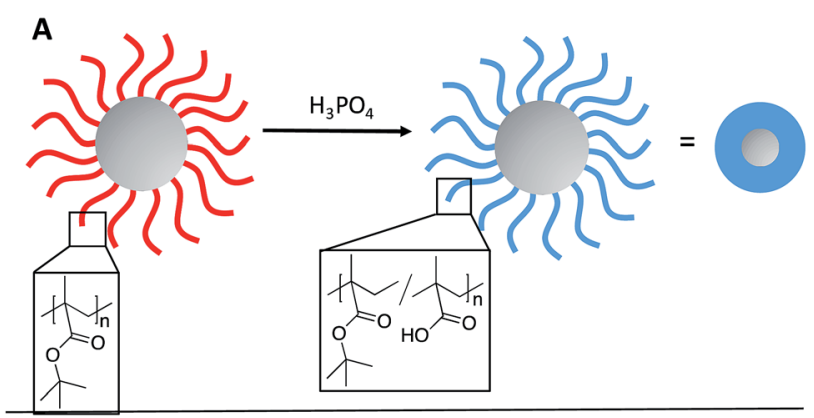

B
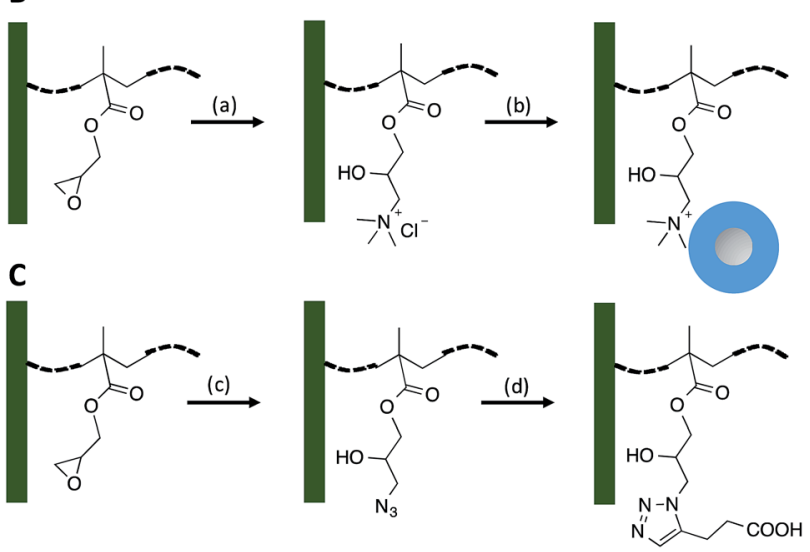

C
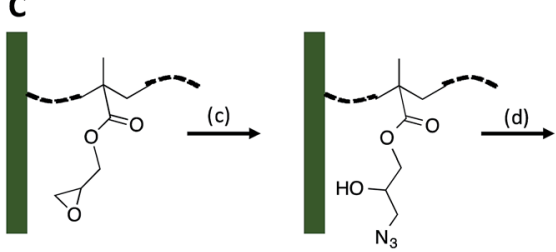

$\mathrm{N}^{\prime \prime}>\mathrm{COOH}$

Fig. 2 Reaction sequences for the synthesis of PMA-SiNP decorated and carboxylic acid functionalized monolith. (A) Polymer brush decorated SiNP; (B) decoration of poly(glycidyl methacrylate-coethylene glycol dimethacrylate) monolith (green) with PMA-SiNP; (C) modification of poly(glycidyl methacrylate-co-ethylene glycol dimethacrylate) monolith with carboxylic acid groups. Reagents: (a) trimethylamine hydrochloride TMA HCl; (b) PMA-SiNP; (c) $\mathrm{NaN}_{3}$; (d) 4pentynoic acid, Cul, PMDETA. 
hydrochloride (TMA $\mathrm{HCl}$ ). Two experimental set-ups were investigated. In the first one the reactant solution was flushed through the monolith at a specific flow rate, concentration, times and temperature. In the second approach the monolith was filled with the reactant solution, sealed and placed in a temperature controlled oven for a definite time. In both cases, a total of $7.21 \times 10^{-5} \mathrm{~mol}$ of GMA in the monolith was assumed per initial concentration in the formulation used to cast the polymeric monolith. TMA $\mathrm{HCl}$ solution at $1.05 \mathrm{M}$, i.e. a large molar excess with respect to the GMA epoxy groups was used ( $\sim 14500$ molar equivalents). It was found that prolonged (24 h) reaction times at $100{ }^{\circ} \mathrm{C}$ are necessary to obtain a homogeneous surface conversion. To monitor the success of this modification steps, the monolith tubes were cut into 5 sections of $3 \mathrm{~mm}$ length and the sections analyzed individually by FTIR (Fig. S1, ESI $\dagger$ ). The results confirm quantitative reaction of the epoxy rings for both methods. When the monolith was treated with a lower concentration of reactant (e.g. $0.5 \mathrm{M}$ in TMA $\mathrm{HCl}$ or $\sim 5000$ molar equivalent with respect to the epoxy ring), irrespective of the approach, the reaction time to reach quantitative and homogenous conversion was prolonged (up to 2 weeks). The observed low reactivity of the epoxy groups (compared to free epoxy groups) is probably because the GMA is part of a less accessible polymer network within a rigid monolith structure.

In the following step the ammonium modified monolith surface was decorated with PMA-SiNP. It was hypothesized that mostly electrostatic interaction would contribute to the trapping of the NP on the monolith surface but frictional forces might play a role as well. Monolith samples were connected to a syringe pump and a suspension of PMA-SiNP flushed through the device. Two important aspects for a successful electrostatic nanoparticle surface decoration of a monolithic material are the suspension flux through the monolith and the NP mass content in the suspension. Indeed, if the suspension flux was excessively high, a NP 'cake' formed at the monolith inlet as the porous structure behaved as a filter. Conversely, if the suspension flux was excessively slow, the time to decorate the surface became impracticably long. Similar problems arose in the case of suspensions with high or low concentrations of PMA-SiNP. Through a number of experiments a PMA-SiNP mass content $0.5 \mathrm{mg} \mathrm{mL}^{-1}$ at a flow rate of $2000 \mu \mathrm{L} \mathrm{h}^{-1}$ were found to produce the best results for a $15 \mathrm{~mm}$ long monolith. To obtain a uniform surface decoration, the flow direction was reversed every $5 \mathrm{~mL}$. The decoration process could easily be monitored visually by the change of the cloudiness of the exiting suspension. Due to the nanoparticles trapping in the monolith the exiting solution was significantly clearer than the injected suspension. When no change in cloudiness was observed anymore, surface saturation was assumed. By comparison with an untreated ammonium modified monolith, a mass increment of 31-32 $\mathrm{mg}$ due to the presence of the PMA NP was measured corresponding to a $c a$. $120 \%$ mass increase. This was consistent over 15 monoliths. The uniformity of the NP layer on the monolith surface was visualized by SEM (Fig. 3). The images of the final NP decorated surface show a homogeneous NP monolayer while the porous structure of the monolith is not affected, as evidenced by the presence of macropores. Most importantly, inspection of SEM
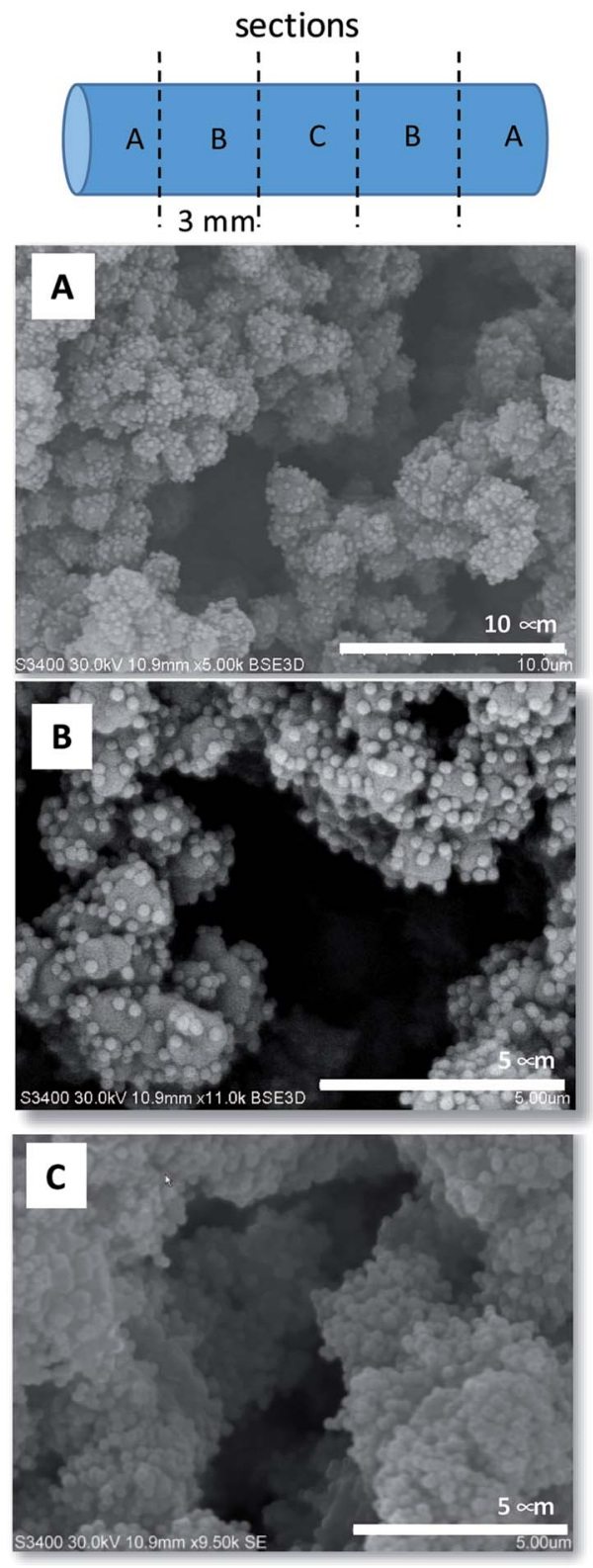

Fig. 3 SEM images taken at different $3 \mathrm{~mm}$ sections of a $15 \mathrm{~mm}$ PMASiNP decorated monolith. Letters in images refer to sections of monolith. Additional images see Fig. S5, ESI. $\dagger$

images of the decorated monolith at different positions (5 sections of $3 \mathrm{~mm}$ ) confirmed a quite uniform PMA-SiNP decoration along the $15 \mathrm{~mm}$ monolith. This result was fully reproducible on three $15 \mathrm{~mm}$ monoliths of the same nature.

The robustness of the electrostatic nanoparticle/surface interactions was monitored by flushing solutions of different pH (4.5-9.5) through the monolith (Table 1). In no case, the mass loss was higher than $4 \%$ and SEM images confirm the unchanged presence of the PMA-SiNP on the monolith surface (Fig. 3). This adhesion robustness can be rationalized by the cooperative nature of the interaction. For each single NP, there is a large number of trapping electrostatic $\mathrm{NP} /$ surface interactions due to the high density of acrylic acid groups in the 
Table $1 \mathrm{pH}$ stability of PMA-SiNP decorated monoliths at a flow rate of $2000 \mu \mathrm{L} \mathrm{h}-1$

\begin{tabular}{llll}
\hline Solution $^{a}$ & $\begin{array}{l}\text { Weight loss (\%) } \\
10 \mathrm{~mL} \text { flushed }\end{array}$ & $\begin{array}{l}\text { Weight loss (\%) } \\
100 \mathrm{~mL} \text { flushed }\end{array}$ & $\begin{array}{l}\text { Solution } \\
\mathrm{pH}\end{array}$ \\
\hline $\mathrm{PBS}$ & 1 & 3 & 7.4 \\
$\mathrm{HCl}$ & 1 & 2 & 4.5 \\
$\mathrm{HCl}$ & 1 & 3 & 6.0 \\
$\mathrm{NaOH}$ & 2 & 4 & 8 \\
$\mathrm{NaOH}$ & 3 & 4 & 9.5 \\
${ }^{a}$ All solutions & $0.1 \mathrm{M}$. & & \\
& & &
\end{tabular}

polymer brush able to prevent the "washing out" of the NP in the presence of competing ions (Fig. 4).

To obtain a comparison material, a bare EDMA-co-GMA monolith was chemically surface-modified with carboxylic acid groups following the reaction sequence in Fig. 2C. In the first step the GMA epoxy groups were reacted with sodium azide. ${ }^{27}$ The success of this reaction is clearly evident from the appearance of an azide signal at $2100 \mathrm{~cm}^{-1}$ in the FTIR spectrum (Fig. S6, ESI †). The second step is a click reaction with 4-pentynoic acid using a suspension of copper(I) iodide (CuI) and $N, N, N^{\prime}, N^{\prime \prime}, N^{\prime \prime}$-penta-methyl-diethylene-triamine (PMDETA) as the catalytic system. Upon click reaction the disappearance of the azide signal is evident from the FTIR spectrum (Fig. S7, ESI†). A yield of $c a$. $70 \%$ was estimated from the spectra and this was reproducible for three identical monoliths.

\section{Comparison of elution capacities of functional monoliths}

Both types of monoliths described above expose the same chemical groups (carboxylic acid) on their surfaces. In one arrangement the carboxylic acid groups are presented as densely coated PMA-SiNP and in the other arrangement they directly cover the monolith surface. Based on the equivalence of the chemical groups, any elution processes will be ruled by the same kind of analyte-solid phase interactions and the elution performance difference can be determined by direct comparison of the material. To investigate that, Myoglobin (M) and Blue Dextran (BD) were selected as analytes. This is rationalized by fact that, while they have a large mass difference (myoglobin $16951 \mathrm{Da}$; blue dextran $2000000 \mathrm{Da}$ ), their isoelectric points are very close (myoglobin, $\mathrm{pI}=7.2 ;^{48}$ blue dextran, $\mathrm{pI}=7.1$ (ref.
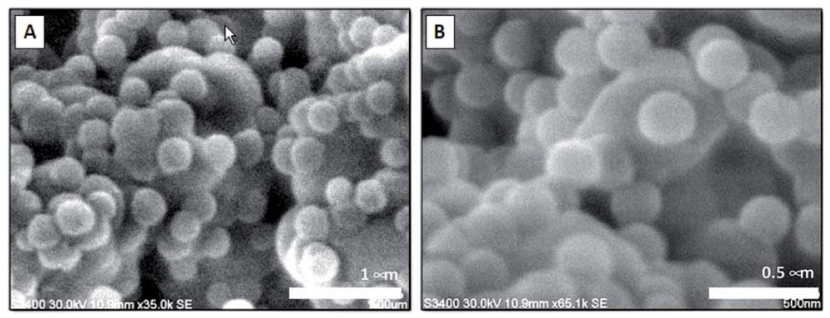

Fig. 4 SEM images of PMA-SiNP decorated monoliths after being flushed with $100 \mathrm{~mL}$ of $0.1 \mathrm{M}$ of PBS (A) and $100 \mathrm{~mL}$ of HCl at $\mathrm{pH}=4.5$ (B). Both images were taken from Section II of the monolith (see Fig. 3).
49)). On this premises, the nature of the analyte-solid phase interactions (typically electrostatic and permanent/induced dipole-dipole interactions) is directly linked to the mass of the analyte as the same PBS buffer solution was used during the elutions. Both buffer pre-conditioned monoliths were separately flushed with myoglobin and blue dextran standard solutions at $C_{0}(\mathrm{M})=C_{0}(\mathrm{BD})=2 \mathrm{mg} \mathrm{mL}^{-1}$, respectively, at a flow rate of $1000 \mu \mathrm{L} \mathrm{h}^{-1}$. Samples were eluted by flushing with aqueous $\mathrm{HCl}$ solution $(\mathrm{pH}=5.4)$ at a flow rate of $1000 \mu \mathrm{L} \mathrm{h}^{-1}$. A set of 50 effluent samples (each with a volume of $0.5 \mathrm{~mL}$ ) was collected and the analyte concentration determined spectroscopically (triplicate error analysis and raw data in Table S1, ESI $\dagger$ ). The experimental data were then fitted with a Boltzmann curve (regression parameters Table S3, ESI $\dagger$ ) to produce the breakthrough curves in Fig. 5. ${ }^{50}$

The PMA-SiNP surface has a strong impact on the elution performances as can be seen from Fig. 5 . While a clean elution of both analytes was achieved with both monoliths, in the case of the PMA-SiNP monolith the elution is shifted to higher retention volumes $\left(V_{\mathrm{R}}\right)$. For example, for the carboxylic acid decorated monolith $V_{\mathrm{R}}=1.73 \mathrm{~mL}$ for myoglobin and $V_{\mathrm{R}}=2.72$
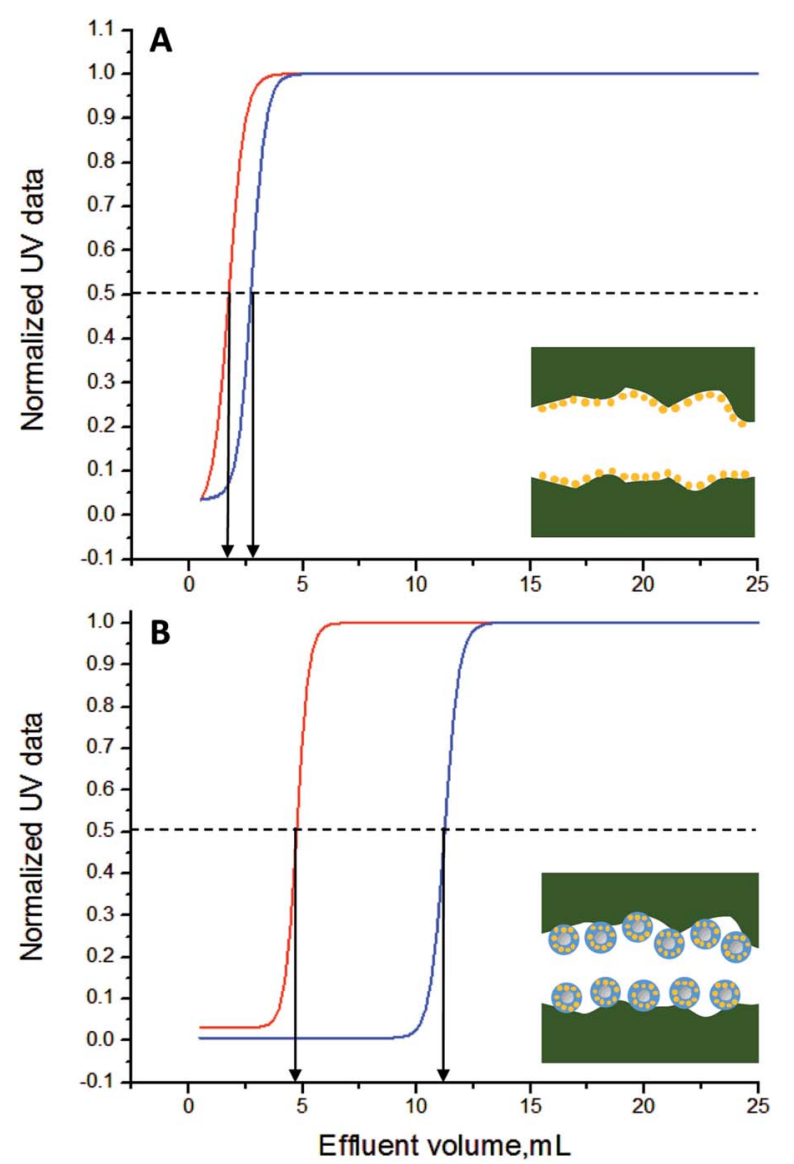

Fig. 5 Best fit curves $\left(R^{2}>0.99\right.$ for all curves) for the extraction performed using myoglobin (in red) and the blue dextran (in blue) for the carboxylic acid functional monoliths (A) and the PMA-SiNP decorated monolith (B). Arrows indicating $V_{R}$. Yellow dots in cartoon represent carboxylic acid functions in surface modified monolith (A) and PMASiNP decorated monolith (B). 
Table 2 Characteristic extraction values for the extraction of Myoglobin (M) and Blue Dextran (BD) on carboxylic acid surface functional (S) and PMA-SiNP (NP) monoliths derived from the four regression curves in Fig. 5 using the equations in Table S2, ESI

\begin{tabular}{llcclll}
\hline Analyte & Monolith & $V_{\mathrm{B}}{ }^{a}(\mathrm{~mL})$ & $V_{\mathrm{R}}{ }^{b}(\mathrm{~mL})$ & $N^{c}$ & $k^{d}$ & $r^{e}(\%)$ \\
\hline $\mathrm{M}$ & $\mathrm{S}$ & 1.2 & 1.7 & 6 & 5 & 53 \\
& $\mathrm{NP}$ & 4.3 & 4.7 & 528 & 527 & 80 \\
$\mathrm{BD}$ & $\mathrm{S}$ & 2.2 & 2.7 & 120 & 119 & 66 \\
& $\mathrm{NP}$ & 10.7 & 11.2 & 2289 & 2288 & 89
\end{tabular}

${ }^{a}$ Breakthrough volume. ${ }^{b}$ Retention volume, outlet concentration $50 \%$ of inlet concentration. ${ }^{c} N$ : theoretical plates. ${ }^{d}$ Retention factor; ratio of analyte adsorbed to solid phase to analyte present in the mobile phase. ${ }^{e}$ Recovery; percentage of analyte that can be recovered.

Table 3 The elution enhancement effects of the four studied SPEs parameters. Each value in this table is the ratio of the respective parameters in Table 2 for surface functional and PMA-SiNP monoliths

\begin{tabular}{lllll}
\hline Analyte & $V_{\mathrm{R}}$ & $N$ & $k$ & $r$ \\
\hline M & 3.7 & 88 & 107 & 1.5 \\
BD & 5.0 & 19 & 19 & 1.4
\end{tabular}

$\mathrm{mL}$ for blue dextran, while it shifts to $4.73 \mathrm{~mL}$ and $11.22 \mathrm{~mL}$ for the PMA-SiNP monolith, respectively. All critical elution parameters are summarized in Table 2. Most notable is the increase in the theoretical plates $(N)$ when going from the simple surface functionalization to the NP system. The relative enhancement from the brush decorated NP monolith in Table 3 was calculated as the ratio of the respective values of the two types of monoliths (Table 2). The elution enhancement cannot solely be explained by the increase in surface area due to the presence of nanoparticles as from geometrical considerations the maximum surface area increase is by factor $\pi$ (Fig. S8, ESI $\dagger$ ) while values in Table 3 are much higher. There is thus a clear contribution of the specific polymer brush. This is supported by the fact that the elution enhancement is not linear but more pronounced for smaller myoglobin, i.e. 88 times increase of $N$ versus 19 for blue dextran. This is coherent with the dimensions of the analytes. The minimum radius of the sphere containing myoglobin is $\sim 25 \mathrm{~nm},{ }^{51}$ which presumably allows a certain extend of diffusion into the $20 \mathrm{~nm}$ PMA brush. The same it is not true for the bigger (and not globular) blue dextran where the minimum radius of the containing sphere is $\sim 125 \mathrm{~nm} .{ }^{51}$ Under the applied conditions the recovery of both analytes was not quantitative, which would need to be further optimized in application development.

\section{Conclusions}

The uniform and dense decoration of polymers monolith with poly(methacrylic acid) brush nanoparticles was achieved. Quantitative analysis of critical extraction parameters for myoglobin and blue dextran confirm an enhancement effect due to the polymer brush on the nanoparticles. While demonstrated here for the elution of biomolecules, the presented proof of concept could be used a platform for the development of extraction devices for other molecules as polymer brushes of a broad spectrum (functional groups, hydrophilicity, etc.) are readily accessible offering the opportunity to tailor monolithic surfaces towards the extraction problem. Moreover, monolith and polymer brushes can be independently optimised, which might overcome practical challenges of monolith functionalisation.

\section{Acknowledgements}

This work was supported by funding from Science Foundation Ireland (07/IN1/B1792). M. I. gratefully acknowledges support from the Biomedical Diagnostic Institute (BDI) and the Irish Separation Science Cluster (ISSC).

\section{Notes and references}

1 I. Nischang, J. Chromatogr. A, 2013, 1287, 39.

2 N. Brun, S. Ungureanu, H. Deleuze and R. Backov, Chem. Soc. Rev., 2011, 40, 771.

3 S. D. Kimmins and N. R. Cameron, Adv. Funct. Mater., 2010, 21, 211.

4 M. S. Silverstein, Prog. Polym. Sci., 2014, 39, 199.

5 C. Viklund, F. Svec, J. M. J. Fréchet and K. Irgum, Chem. Mater., 1999, 8, 744.

6 A. Premstaller, H. Oberacher and C. G. Huber, Anal. Chem., 2000, 72, 4386.

7 A. I. Cooper and A. B. Holmes, Adv. Mater., 1999, 11, 1270.

8 F. Svec, J. Chromatogr. A, 2010, 1217, 902.

9 F. Svec, J. Sep. Sci., 2004, 27, 747.

10 S. Choudhury, D. Connolly and B. White, Anal. Methods, 2015, 7, 6967.

11 E. G. Vlakh and T. B. Tennikova, J. Sep. Sci., 2007, 30, 2801.

12 F. Svec, J. Sep. Sci., 2004, 27, 1419.

13 N. Smith and Z. Jiang, J. Chromatogr. A, 2008, 1184, 416.

14 G. Guichon, J. Chromatogr. A, 2007, 1168, 101.

15 F. Svec and J. M. J. Frechet, Anal. Chem., 1992, 64, 820.

16 R. Mallik and D. S. Hage, J. Sep. Sci., 2006, 29, 1686.

17 F. Svec, J. Chromatogr. A, 2012, 1228, 250.

18 I. Nischang, I. Teasdale and O. Brueggemann, Anal. Bioanal. Chem., 2011, 400, 2289.

19 F. Svec, Electrophoresis, 2006, 27, 947.

20 A. Nordberg and E. F. Hilder, Anal. Bioanal. Chem., 2009, 394, 71.

21 F. Svec and A. A. Kurganov, J. Chromatogr. A, 2008, 1184, 281. 22 F. Svec, J. Chromatogr. B: Anal. Technol. Biomed. Life Sci., 2006, 841, 52.

23 O. Potter and E. F. Hilder, J. Sep. Sci., 2008, 31, 1881.

24 J. O. Zoppe, N. C. Ataman, P. Mocny, J. Wang, J. Moraes and H.-A. Klok, Chem. Rev., 2017, 117, 1105.

25 T. Rohr, E. F. Hilder, J. J. Donovan, F. Svec and J. M. J. Fréchet, Macromolecules, 2003, 36, 1677.

26 J. Krenkova, A. Gargano, N. A. Lacher, J. M. Schneiderheinze and F. Svec, J. Chromatogr. A, 2009, 1216, 6824.

27 L. Moine, H. Deleuze, M. Degueil and B. Maillard, J. Polym. Sci., Part A: Polym. Chem., 2004, 42, 1216. 
28 D. Cummins, P. Wyman, C. J. Duxbury, J. Thies, C. E. Koning and A. Heise, Chem. Mater., 2007, 19, 5285.

29 F. Audouin, R. Larragy, M. Fox, B. O'Connor and A. Heise, Biomacromolecules, 2012, 13, 3787.

30 D. Cummins, C. J. Duxbury, P. J. L. M. Quaedflieg, P. C. M. M. Magusin, C. E. Koning and A. Heise, Soft Matter, 2009, 5, 804.

31 R. Zhang, G. Yang, P. Xin, L. Qi and Y. Chen, J. Chromatogr. A, 2009, 1216, 2404.

32 F. Audouin and A. Heise, Eur. Polym. J., 2013, 49, 1073.

33 F. Audouin, M. Fox, R. Larragy, P. Clarke, J. Huang, B. O'Connor and A. Heise, Macromolecules, 2012, 45, 6127.

34 W. W. Hu, T. T. Hong, X. Gao and Y. Ji, Trends Anal. Chem., 2014, 61, 29.

35 N. N. Liang and B. Zhang, Comb. Chem. High Throughput Screening, 2011, 14, 182.

36 A.-H. Duan, S.-H. Xie and L.-M. Yuan, Trends Anal. Chem., 2011, 30, 484.

37 E. P. Nesterenko, P. N. Nesterenko, D. Connolly, X. He, P. Floris, E. Duffy and B. Paull, Analyst, 2013, 138, 4229.

38 D. Connolly, S. Currivan and B. Paull, Proteomics, 2012, 12, 2904.
39 S. S. Tong, S. X. Liu, H. Q. Wang and Q. Jia, Chromatographia, 2104, 77, 5 .

40 E. F. Hilder, F. Svec and J. M. J. Frechet, J. Chromatogr. A, 2004, 1053, 101.

41 J. P. Hutchinson, P. Zakaria, A. R. Bowie, M. Macka, N. Avdalovic and P. R. Haddad, Anal. Chem., 2005, 77, 407.

42 J. P. Hutchinson, E. F. Hilder, R. A. Shellie, J. A. Smith and P. R. Haddad, Analyst, 2006, 131, 215.

43 P. Zakaria, J. P. Hutchinson, N. Avdalovic, Y. Liu and P. R. Haddad, Anal. Chem., 2005, 77, 417.

44 J. P. Hutchinson, E. F. Hilder, M. Macka, N. Avdalovic and P. R. Haddad, J. Chromatogr. A, 2006, 1109, 10.

45 M. Iacono, D. Connolly and A. Heise, Materials, 2016, 9, 263. 46 K. Matyjaszewski, H. Dong, W. Jakubowski, J. Pietrasi and A. Kusumo, Langmuir, 2007, 23, 4528.

47 M. Iacono and A. Heise, Polymers, 2015, 7, 1427.

48 www.sigmaaldrich.com/catalog/product/sigma/m9267?

lang $=$ fr\&region $=F R$, accessed 06.07.2013.

49 J. R. L. Walker, Biochem. Educ., 1992, 20, 42.

50 E. Bacalum, M. Radulescu, E. E. Iorgulescu and V. David, Rev. Roum. Chim., 2011, 56, 137.

51 H. P. Erickson, Biol. Proced. Online, 2009, 11, 32. 